\title{
Low-redshift lowest-metallicity star-forming galaxies in the SDSS DR14
}

\author{
Y. I. Izotov ${ }^{1,2}$, N. G. Guseva ${ }^{1,2}$, K. J. Fricke ${ }^{1,3}$, and C. Henkel ${ }^{1,4}$ \\ 1 Max-Planck-Institut für Radioastronomie, Auf dem Hügel 69, 53121 Bonn, Germany \\ 2 Bogolyubov Institute for Theoretical Physics, National Academy of Sciences of Ukraine, 14-b Metrolohichna str., \\ Kyiv 03143, Ukraine \\ e-mail: yizotov@itp.kiev.ua \\ 3 Institut für Astrophysik, Göttingen Universität, Friedrich-Hund-Platz 1, 37077 Göttingen, Germany \\ 4 Astronomy Department, King Abdulaziz University, PO Box 80203, Jeddah 21589, Saudi Arabia
}

Received 3 December 2018 / Accepted 16 January 2019

\begin{abstract}
We present a sample of low-redshift $(z<0.133)$ candidates for extremely low-metallicity star-forming galaxies with oxygen abundances $12+\log \mathrm{O} / \mathrm{H}<7.4$ selected from the Data Release 14 (DR14) of the Sloan Digital Sky Survey (SDSS). Three methods are used to derive their oxygen abundances. Among these methods two are based on strong [O II] $] 3727 \AA$, [O III] $] \lambda 4959 \AA$, and [O III] $\lambda 5007 \AA$ emission lines, which we call strong-line and semi-empirical methods. These were applied for all galaxies. We have developed one of these methods, the strong-line method, in this paper. This method is specifically focused on the accurate determination of metallicity in extremely low-metallicity galaxies and may not be used at higher metallicities with $12+\log \mathrm{O} / \mathrm{H} \gtrsim 7.5$. The third, the direct $T_{\mathrm{e}}$ method, was applied for galaxies with detected [O III] $\lambda 4363$ emission lines. All three methods give consistent abundances and can be used in combination or separately for selection of lowest-metallicity candidates. However, the strong-line method is preferable for spectra with a poorly detected or undetected [O $\mathrm{III}] \lambda 4363$ emission line. In total, our list of selected candidates for extremely low-metallicity galaxies includes 66 objects.
\end{abstract}

Key words. galaxies: abundances - galaxies: irregular - galaxies: evolution - galaxies: formation - galaxies: ISM - HII regions

\section{Introduction}

Nearby dwarf star-forming galaxies (SFG) with extremely low metallicities are often considered as local counterparts of highredshift galaxies. Their proximity allows us to study them in much greater detail than the high-redshift galaxies and to establish useful constraints on the physical conditions of their low-metallicity interstellar medium (ISM), the origin of chemical elements, and to develop models of stellar evolution. These studies can be used to analyse the physical properties of the primeval galaxies at redshifts $z \sim 5-10$, which are thought to be dwarf systems responsible for the reionization of the Universe (Ouchi 2009; Wise \& Cen 2009; Bouwens et al. 2015; Robertson 2013; Robertson et al. 2015; Khaire et al. 2016).

However, the number of known nearby lowest-metallicity SFGs with oxygen abundances $12+\log \mathrm{O} / \mathrm{H} \lesssim 7.35$ is very low. Among them are SFGs with $12+\log \mathrm{O} / \mathrm{H} \sim 7.00$. These are $\mathrm{J} 0811+4730$ with $12+\log \mathrm{O} / \mathrm{H}=6.98 \pm 0.02$ (Izotov et al. 2018a), A198691 with $12+\log \mathrm{O} / \mathrm{H}=7.02 \pm$ 0.03 (Hirschauer et al. 2016) and SBS 0335-052W with $12+$ $\log \mathrm{O} / \mathrm{H}$ ranging from 6.86 to 7.22 in different star-forming regions (Izotov et al. 2009). Recently, Annibali (2019) reported the range 6.96-7.14 in different $\mathrm{H}$ II regions of DDO 68. Additionally, Pustilnik et al. (2016) and Kniazev et al. (2018) derived $12+\log \mathrm{O} / \mathrm{H} \lesssim 7.35$ in nearly a dozen of galaxies in the Lynx-Cancer and Eridanus voids.

Large spectroscopic surveys, such as the Sloan Digital Sky Survey (SDSS) containing millions of galaxy spectra in its database, open an opportunity to considerably increase the sample of the extremely low-metallicity SFGs with $12+$ $\log \mathrm{O} / \mathrm{H} \lesssim 7.35$ (e.g. Izotov et al. 2012; Guseva et al. 2017). In particular, the SFG with a record low luminosity-weighted metallicity, J0811+4730, was found in the SDSS.

The empirical diagnostic [O III] $\lambda 5007 / \mathrm{H} \beta-[\mathrm{N}$ II $] \lambda 6584 / \mathrm{H} \alpha$ diagram (Baldwin et al. 1981) can successfully be used to preselect the candidates to extremely low-metallicity SFGs. On this diagram, they occupy the region with both very low $\left[\begin{array}{ll}\mathrm{III}\end{array}\right] \lambda 5007 / \mathrm{H} \beta \leq 3$ and very low $[\mathrm{N}$ II $] \lambda 6584 / \mathrm{H} \alpha \leq 0.1$, far from the main sequence of low-redshift SFGs (Izotov et al. 2012). Similarly, the empirical $\mathrm{R}_{23}-\mathrm{O}_{32}$ diagram can be used, where $\mathrm{R}_{23}=\left(\left[\begin{array}{ll}\mathrm{O} & \text { II }\end{array}\right] \lambda 3727+\left[\begin{array}{ll}\mathrm{O} & \text { III }\end{array}\right] \lambda 4959+\left[\begin{array}{ll}\mathrm{O} & \text { III }\end{array}\right] \lambda 5007 / \mathrm{H} \beta\right.$ and $\mathrm{O}_{32}=[\mathrm{O}$ III $] \lambda 5007 /[\mathrm{O}$ II $] \lambda 3727$ (Izotov et al. 2018a).

A variety of methods have been developed and can be used for the abundance determination. The most reliable method is the direct method, but it requires the detection, with good accuracy, of the $[\mathrm{O}$ III] $\lambda 4363$ emission line for the electron temperature determination. For SFGs with weak or undetected [O III] $] 4363$ emission lines only strong-line methods can be used. These methods are based on combinations of strong emission-line intensities of various elements, such as O, N, S, and Ar. However, the problem with strong-line methods is that the intensities of emission lines depend not only on the metallicity but also on some other quantities, such as the ionization parameter. Many of these methods were constructed for application to galaxies in a wide range of metallicities, typically at oxygen abundances $12+\log \mathrm{O} / \mathrm{H} \gtrsim 7.4$, and often on expence of the accuracy in the abundance determination at lowest metallicities (e.g. Pilyugin \& Thuan 2005; Pilyugin \& Grebel 2016; 
Nagao et al. 2006; Yin et al. 2007). This paper focuses on simple empirical strong-line methods for extremely low-metallicity objects based on oxygen lines. Emphasizing systems with abundances much lower than solar, we aim to increase the accuracy of abundance determinations in SFGs with undetected or weak [O III] $\lambda 4363$ emission. The construction of this method is also motivated by the fact that recently a number of new extremely low-metallicity SFGs have been discovered for which the oxygen abundances were derived using high-quality spectra (Izotov et al. 2018a, and references therein) allowing for more reliable calibration with the direct method at lowest metallicities. The new method is applied in the present paper to search for extremely low-metallicity candidates in the SDSS Data Release 14.

The data base used for the selection of the lowest-metallicity SFGs is described in Sect. 2. Methods of the oxygen abundance determination are discussed in Sect. 3. The results of the abundance determination and the list of the SFGs with $12+\log \mathrm{O} / \mathrm{H}<$ 7.4 are presented in Sect. 4. The application of diagnostic diagrams for the selection of extremely low-metallicity SFGs is considered in Sect. 5. Our main conclusions are summarised in Sect. 6.

\section{Data}

We have searched for extremely low-metallicity galaxies from a sample of $\sim 30000$ SFGs selected from the spectroscopic database of the SDSS DR14 (Abolfathi et al. 2018). This is a continuation of studies which used earlier SDSS Data Releases and were published by Izotov et al. (2012) and Guseva et al. (2015, 2017). Details of SDSS sample selection can be found in Izotov et al. (2014a). This sample includes SFGs with equivalent widths $\operatorname{EW}(\mathrm{H} \beta) \gtrsim 10 \AA$ of the $\mathrm{H} \beta$ emission line in their spectra indicating an active star formation and the presence of hot massive stars. The [O III] $\lambda 4363 \AA$ emission line is present in spectra of $\sim 18700$ out of $\sim 30000$ SFGs. In $~ 2000$ SDSS spectra this line is measured with an accuracy better than $25 \%$ allowing for the reliable determination of element abundances, most often of oxygen, neon and nitrogen.

The line fluxes and their errors in each spectrum were measured using the IRAF $^{1}$ splot routine and then corrected for extinction. The internal extinction was derived from the Balmer hydrogen emission line fluxes after correction for the Milky Way extinction. The line fluxes were corrected for both reddening (Cardelli et al. 1989) and underlying hydrogen stellar absorption by the application of an iterative procedure (Izotov et al. 1994) and were used for the element abundance determination. The same SDSS spectra were used for the determination of some integrated characteristics such as $\mathrm{H} \beta$ luminosities, $L(\mathrm{H} \beta)$, star formation rates SFR, and stellar masses $M_{\star}$, adopting a luminosity distance derived with a cosmological calculator (NED, Wright 2006), based on the cosmological parameters $\mathrm{H}_{0}=67.1 \mathrm{~km} \mathrm{~s}^{-1} \mathrm{Mpc}^{-1}, \Omega_{\Lambda}=0.682$, and $\Omega_{m}=0.318$ (Planck Collaboration XV 2014).

\section{Methods of oxygen abundance determinations}

One of the commonly used methods of the oxygen abundance determination in low-metallicity SFGs is the direct method based

\footnotetext{
1 IRAF is the Image Reduction and Analysis Facility distributed by the National Optical Astronomy Observatory, which is operated by the Association of Universities for Research in Astronomy (AURA) under cooperative agreement with the National Science Foundation (NSF).
}

on the determination of the electron temperature $T_{\mathrm{e}}(\mathrm{O}$ III $)$ from the $[\mathrm{O}$ III] $\lambda 4363 /(\lambda 4959+\lambda 5007)$ emission-line ratio and on the relation between $T_{\mathrm{e}}(\mathrm{O}$ II $)$ and $T_{\mathrm{e}}(\mathrm{O}$ III $)$ obtained by, for example, Izotov et al. (2006a) from the photoionized $\mathrm{H}$ II region models. The knowledge of the temperatures and the presence of [O II] and [O III] emission lines in the SDSS spectra allowed us to determine the abundances of these two most abundant oxygen ions in the $\mathrm{H}$ II regions and thus the total oxygen abundances for a large sample of SFGs. However, the [O III] $\lambda 4363 \AA$ emission line is weak in most SDSS spectra of low-metallicity SFGs and is detected with low signal-to-noise ratio introducing large uncertainties in the determination of the electron temperature and oxygen abundance.

Therefore, for these galaxies, other methods based on strong emission lines are needed to more reliably derive their metallicities. In this paper we have developed a purely empirical strong-line method based on the SDSS DR14 sample aiming to apply it for selection of the extremely low-metallicity SFG candidates. This method uses the spectroscopic properties of well-studied lowest-metallicity galaxies and is calibrated with the direct method. For the strong-line method the most natural is to use the emission-line fluxes of oxygen, the most abundant heavy element. The common approach in the past was to use the relation between the metallicity and the sum of the fluxes of strong oxygen lines in the optical range, $\mathrm{R}_{23}=\left(\left[\mathrm{O}_{\text {III }}\right] \lambda 3727+\left[\mathrm{O}_{\text {III }}\right] \lambda 4959+\left[\mathrm{O}_{\text {III }}\right] \lambda 5007\right) / \mathrm{H} \beta$.

The problem with this method is that the relation between the metallicity and $\mathrm{R}_{23}$ consists of low- $(12+\log \mathrm{O} / \mathrm{H} \lesssim 8.0)$ and high-metallicity $(12+\log \mathrm{O} / \mathrm{H} \gtrsim 8.0)$ branches and thus the same value of $\mathrm{R}_{23}$ corresponds to two metallicities (e.g. Pagel et al. 1980; Edmunds \& Pagel 1984; McGaugh 1991). Therefore, other constraints separating low- and high-metallicity branches are needed to resolve this ambiguity. One of the possible solutions is to use the relation between the metallicities and [N II] $] 6584 / \mathrm{H} \alpha$ emission line flux ratios which monotonically increase with metallicity and thus this relation is not degenerate (e.g. van Zee et al. 1998; Pettini \& Pagel 2004). However, the use of this relation for the determination of the oxygen abundances is limited because the [N $\mathrm{NI}] \lambda 6584$ emission line is very weak or undetected in low-metallicity galaxies with high-excitation $\mathrm{H}$ II regions. We have used this line (or the upper limit of its flux) to separate low- and high-metallicity branches. Adopting [ $\left.\mathrm{N}_{\mathrm{II}}\right] \lambda 6584 / \mathrm{H} \beta \lesssim 0.2$ would then select objects located on the low-metallicity branch of the $\log R_{23}-$ $12+\log \mathrm{O} / \mathrm{H}$ relation.

To construct the $\log \mathrm{R}_{23}-12+\log \mathrm{O} / \mathrm{H}$ diagram we select $\sim 2000$ SDSS DR14 SFGs in which the [O III] $\lambda 4363$ emission line is detected with an accuracy better than $25 \%$ (see Sect. 2) allowing for a reliable oxygen abundance determination (grey dots in Fig. 1a). Additionally, all these galaxies show in their spectra [O II] $] 33727 \AA,[\mathrm{O}$ III] $\lambda 4959 \AA, \lambda 5007 \AA$ and have $[\mathrm{O}$ III] $\lambda 4959 / \mathrm{H} \beta \geq 0.2$. These data are supplemented by various samples of SFGs with reliably detected [O III] $\lambda 4363 \AA$ emission lines in their spectra: a sample of extremely low-metallicity SFGs with $12+\log \mathrm{O} / \mathrm{H} \leq 7.35$ (filled symbols in Fig. 1a; Hsyu et al. 2017; Annibali 2019; Izotov et al. 2018a, and references therein), a sample of SFGs with highest $\mathrm{O}_{32}=[\mathrm{O}$ III $] \lambda 5007 /[\mathrm{O}$ II $] \lambda 3727$ ratios of $\sim 20-40$ (asterisks in Fig. 1a; Izotov et al. 2017, and references therein), a sample of Lyman continuum leaking galaxies with $\mathrm{O}_{32}$ ratios in the range $\sim 5-28$ (crosses in Fig. 1a; Izotov $2018 \mathrm{~b}$, and references therein), and a sample of SFGs used for the primordial $\mathrm{He}$ abundance determination (open circles in Fig. 1a; Izotov et al. 2014b, and references therein). We note that 

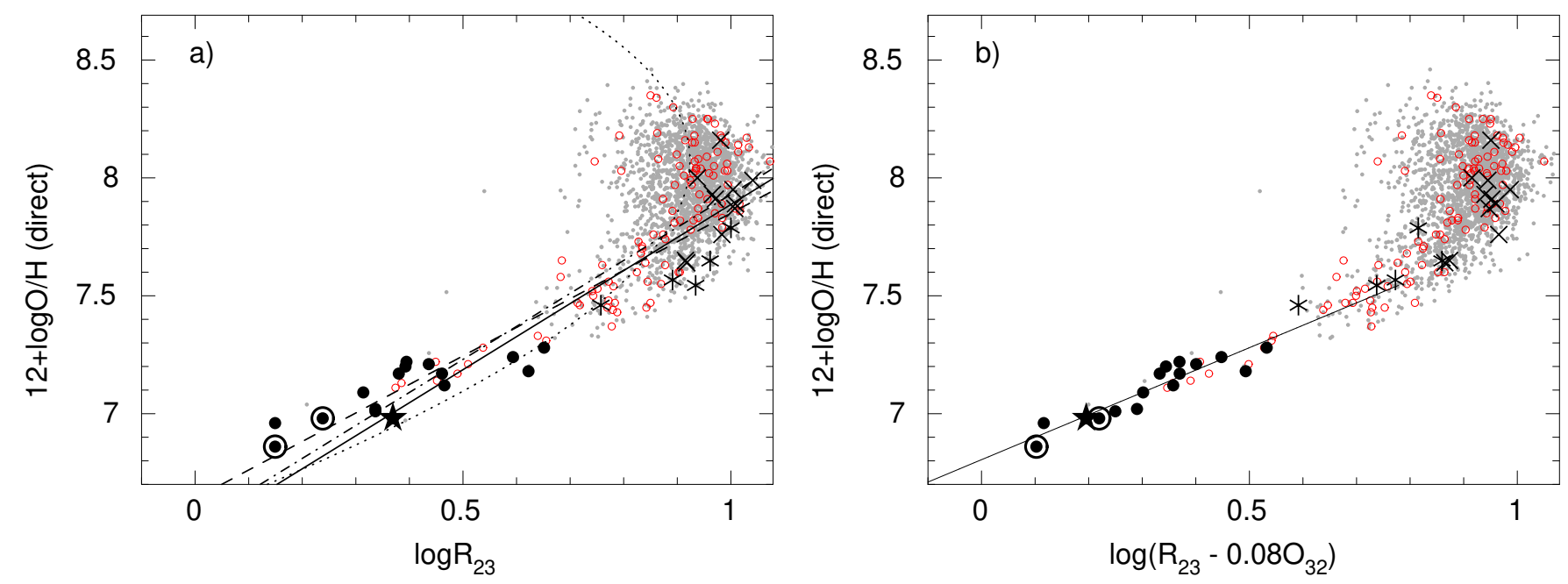

Fig. 1. Panel a: $\left(\log \mathrm{R}_{23}\right)-(12+\log \mathrm{O} / \mathrm{H})$, and panel $b$ : $\log \left(\mathrm{R}_{23}-0.08 \mathrm{O}_{32}\right)-(12+\log \mathrm{O} / \mathrm{H})$ relations. In both these relations $12+\log \mathrm{O} / \mathrm{H}$ is derived by the direct method, $\mathrm{R}_{23}=\left([\mathrm{O}\right.$ III $\left.] \lambda 4959+\lambda 5007+\left[\mathrm{O}_{\mathrm{II}}\right] \lambda 3727\right) / \mathrm{H} \beta$ and $\mathrm{O}_{32}=\left[\mathrm{O}_{\mathrm{III}}\right] \lambda 5007 /[\mathrm{O}$ II $] \lambda 3727$. The galaxy J0811+4730 with the lowest luminosity-weighted oxygen abundance (Izotov et al. 2018a) is shown by a filled star, while two knots in SBS 0335-052W with similar or lower oxygen abundances (Izotov et al. 2009) are represented by encircled filled circles. Other lowest-metallicity SFGs from Izotov et al. (2018a), the galaxy Little Cub (Hsyu et al. 2017) and the $\mathrm{H}$ in region DDO 68 \#7 (Annibali 2019) are shown by filled circles. SFGs with the highest $\mathrm{O}_{32}$ ratios in the range 20-40 (Izotov et al. 2017) are shown by asterisks. Samples of SFGs used for the He abundance determination (Izotov et al. 2014a, and references therein) and Lyman continuum leakers (Izotov 2018b, and references therein) are shown by open circles and crosses, respectively. Grey dots represent SDSS SFGs at $z>0.02$ from DR9 and earlier releases and at any redshift from DR10 and later releases, with the [O III] $\lambda 4363 \AA$ emission line measured with an accuracy better than $25 \%$. The dashed, dotted, solid, and dash-dotted lines in panel a are the empirical relations by Skillman (1989), Nagao et al. (2006), Yin et al. (2007) and Pilyugin (2000), respectively. The solid line in panel $b$ is the linear most likelihood fit to the SDSS data with $\log \left(\mathrm{R}_{23}-0.08 \mathrm{O}_{32}\right) \leq 0.8$ (this paper).

two encircled filled circles indicate the location of star-forming regions 3 and 4 in SBS 0335-052W (Izotov et al. 2009) with undetected [O III] $\lambda 4363 \AA$ line. They are shown because of their extremely low oxygen abundances, which are among the lowest known and considerably lower than the luminosity-weighted oxygen abundance 7.12 of SBS 0335-052W (Izotov \& Thuan 2007). The electron temperatures and the oxygen abundances in these objects are derived by Izotov \& Thuan (2007) using the semiempirical method. However, we have not used them in our subsequent fitting of relations for the abundance determination. It is also worth to mention SFG J0811+4730 because of showing the lowest luminosity-weighted oxygen abundance $12+\log \mathrm{O} / \mathrm{H}=$ $6.98 \pm 0.02$ known so far (filled star in Fig. 1a; Izotov et al. 2018a).

It is important that the data include the objects with the highest $\mathrm{O}_{32}$ which are indicators of a very high ionization parameter $U$ that is the measure of the number of ionizing photons per one ion in the $\mathrm{H}$ II region. The use of these galaxies allows us to eliminate the dependence of the calibration relation on $U$.

For the sake of comparison, we show in Fig. 1a some simple calibration relations produced in the past. Three of them, those by Skillman (1989), Pilyugin (2000) and Yin et al. (2007), are linear. We note that these relations reproduce the oxygen abundances reasonably well (within $\sim 0.2 \mathrm{dex}$ ) of the extremely low-metallicity SFGs with $\log \mathrm{R}_{23}<0.5$ shown by filled symbols. On the other hand, SFGs with highest $\mathrm{O}_{32}$ at $\log \mathrm{R}_{23}>0.5$ (asterisks and three SFGs shown by filled circles) considerably deviate from both the SDSS SFGs and linear relations.

We note the relatively high scatter of filled symbols in the diagram which we attribute to the ionization parameter varying in a large range. To minimize the scatter caused by various ionization parameters we show in Fig. $1 \mathrm{~b}$ the relation $\log \left(\mathrm{R}_{23}-0.08 \mathrm{O}_{32}\right)-12+\log \mathrm{O} / \mathrm{H}$. The distributions of SFGs in
Fig. 1b show a much lower scatter than in Fig. 1a. The data in Fig. $1 \mathrm{~b}$ can be reproduced by the linear relation

$12+\log \frac{\mathrm{O}}{\mathrm{H}}=0.950 \log \left(\mathrm{R}_{23}-0.08 \mathrm{O}_{32}\right)+6.805$,

shown by a solid line.

\section{Selection of candidates to the extremely low-metallicity SFGs in SDSS DR14}

We applied Eq. (1) for selection of candidates to the extremely low-metallicity SFGs with $12+\log \mathrm{O} / \mathrm{H}<7.4$. It is seen in Fig. 1b that these low metallicities correspond to $\left(\mathrm{R}_{23}-0.08 \mathrm{O}_{32}\right) \lesssim 4$. We also adopted an upper limit of $[\mathrm{N}$ II $] \lambda 6584 / \mathrm{H} \beta \leq 0.2$ to exclude the contamination of the sample from objects on the upper branch of the $\log \mathrm{R}_{23}-12+\log \mathrm{O} / \mathrm{H}$ relation, and put a low limit [O III $] \lambda 4959 / \mathrm{H} \beta \gtrsim 0.2$ to exclude the galaxies which were not tested in Sect. 3 with the direct method because of the very weak [O III] $\lambda 4363 \AA$ emission line.

The [O II] $\lambda 3727 \AA$, [O III] $\lambda 4959 \AA, \lambda 5007 \AA$ emission line intensities are needed to derive $12+\log \mathrm{O} / \mathrm{H}$ by the strongline method discussed in Sect. 3. However, most of SDSS lowest-metallicity galaxies are at low redshifts. Therefore, the [O II] $\lambda 3727 \AA$ emission line is outside the wavelength range of $\sim 3800-9200 \AA$ in spectra of galaxies with $z \lesssim 0.02$ selected in DR9 and earlier releases, precluding the determination of metallicity while spectra of DR10 and later releases are obtained in a larger wavelength range of $~ 3600-10300 \AA$ including the rest-frame wavelength of [O II $] \lambda 3727 \AA$ emission line. To avoid this difficulty with spectra of DR9 and earlier releases some prescriptions are needed to estimate the intensity of the [O II] $] 3727 \AA$ emission line from intensities of other lines. In particular, Izotov et al. (2012) and Guseva et al. (2015, 2017) 

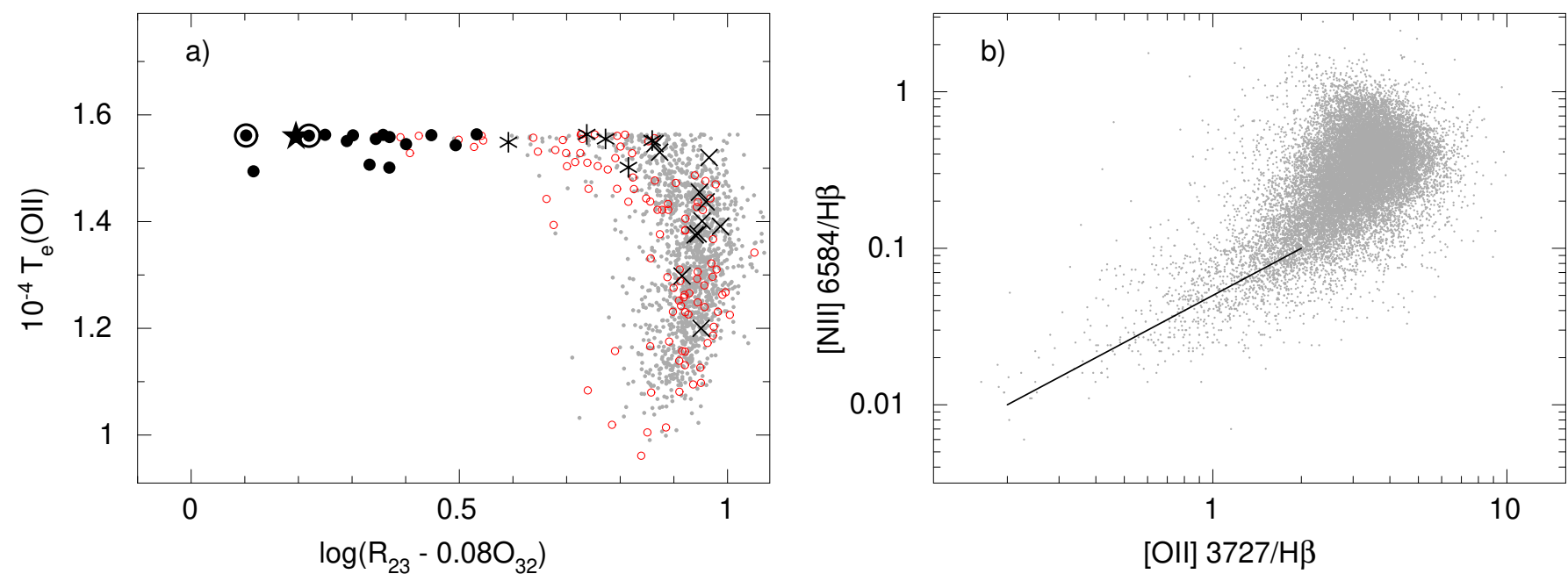

Fig. 2. Panel $a: \log \left(\mathrm{R}_{23}-0.08 \mathrm{O}_{32}\right)-\mathrm{T}_{\mathrm{e}}(\mathrm{O}$ II) relation for SFGs. Panel $b$ : relation between the extinction-corrected [O II] $] 3727 / \mathrm{H} \beta$ and $[\mathrm{N}$ II $] \lambda 6584 / \mathrm{H} \beta$ flux ratios. The solid line corresponds to $[\mathrm{O}$ II $] \lambda 3727=20 \times[\mathrm{N}$ II $] \lambda 6584$. Symbols in both panels are as in Fig. 1 .

used for that [O II] $\lambda 7320 \AA, \lambda 7330 \AA$ emission lines. However, these lines are relatively weak or undetected introducing large uncertainties. Additionally, for galaxies at redshifts $z \sim 0.02-$ 0.03 , their observed wavelengths are close to the wavelength $\sim 7500 \AA$ of strong telluric absorption. Keeping in mind these caveats, we decide to use the $[\mathrm{O}$ II $] \lambda 7320 \AA, \lambda 7330 \AA$ emission lines if detected for the determination of the [O II $] \lambda 3727 \AA$ emission line flux from the relation (Aller 1984; Izotov et al. 2006a)

$\log I([\mathrm{O} \mathrm{II}] \lambda 3727)=0.961+\frac{8110}{T_{\mathrm{e}}(\mathrm{O} \text { II })}+\log I([\mathrm{O} \mathrm{II}] \lambda 7325)$,

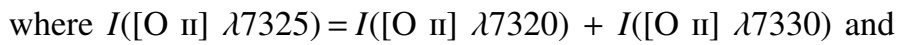
$T_{\mathrm{e}}\left(\mathrm{O}\right.$ II) is the electron temperature in the $\mathrm{O}^{+}$zone of the $\mathrm{H} \mathrm{II}_{\text {II }}$ region from where $[\mathrm{O}$ II] emission lines originate. Thus the elec-

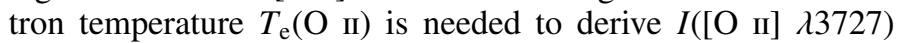
using Eq. (2).

There is no need to use Eq. (2) if [O II] $\lambda 3727 \AA$ is observed. If it is not observed but [O III] $] 4363 \AA$ is detected, then both $T_{\mathrm{e}}(\mathrm{O}$ III $)$ and $T_{\mathrm{e}}(\mathrm{O}$ II) can be derived. The most complicated case for SDSS spectra of low-metallicity galaxies is when both $[\mathrm{O}$ II] $] \lambda 3727 \AA$ and [O III] $] \lambda 4363 \AA$ A emission lines are not detected. Then some estimates of the electron temperature $T_{\mathrm{e}}(\mathrm{O}$ II) are needed to use the Eq. (2). To do that we consider the relation between $T_{\mathrm{e}}(\mathrm{O}$ II $)$ and $\log \left(\mathrm{R}_{23}-0.08 \mathrm{O}_{32}\right)$ for the galaxies with spectra where the $\left[\mathrm{O}_{\text {II }}\right] \lambda 3727 \AA$ line is present and the $[\mathrm{O}$ III] $\lambda 4363 \AA$ line is detected with a relative error better than $25 \%$. This allowed us to derive both $T_{\mathrm{e}}(\mathrm{O}$ II) with good accuracy and $R_{23}-0.08 O_{32}$. The relation is shown in Fig. 2a. It is seen that $T_{\mathrm{e}}\left(\mathrm{O}\right.$ II) at high $\mathrm{R}_{23}-0.08 \mathrm{O}_{32}$ varies in a wide range, but it is nearly constant with the value of $\sim 15500 \mathrm{~K}$ at lower $\mathrm{R}_{23}-0.08 \mathrm{O}_{32}$. This constancy is due to the fact that $T_{\mathrm{e}}(\mathrm{O}$ III $)$ at low $\mathrm{R}_{23}-0.08 \mathrm{O}_{32}$ asymptotically approaches the value $\sim 22000 \mathrm{~K}$. It is also worth to note that cLoudy photoionization models of hottest $\mathrm{H}$ II regions predict $T_{\mathrm{e}}(\mathrm{O}$ III $)$ and $T_{\mathrm{e}}(\mathrm{O}$ II) of $\sim 22000 \mathrm{~K}-24000 \mathrm{~K}$ and $\sim 15500 \mathrm{~K}$, respectively (Ferland et al. 1998; Ferland 2013). We adopt the value $T_{\mathrm{e}}\left(\mathrm{O}_{\mathrm{II}}\right)=15500 \mathrm{~K}$ for the determination of $I([\mathrm{O}$ II] 23727$)$ in the lowest-metallicity galaxy candidates for which the condition $\mathrm{R}_{23}-0.08 \mathrm{O}_{32}<5$ is satisfied corresponding to $12+\log \mathrm{O} / \mathrm{H} \lesssim 7.5$.

We used the extinction-corrected [ $\mathrm{N}$ II] $\lambda 6584$ emission line to estimate the $[\mathrm{O}$ II] $\lambda 3727$ emission-line flux for objects with undetected $[\mathrm{O}$ I $] \lambda 7325$ emission in their spectra. To find the relation between $[\mathrm{N}$ II] $] 6584$ and $[\mathrm{O}$ II] $] 3727$ fluxes we show in Fig. $2 \mathrm{~b}$ the diagram $[\mathrm{N}$ II] $] \lambda 6584 / \mathrm{H} \beta-[\mathrm{O}$ II $] \lambda 3727 / \mathrm{H} \beta$ for SDSS DR14 SFG spectra with detected [N II] $\lambda 6584$ and [O II] $] 3727$ lines. We see that

$[\mathrm{OII}] \lambda 3727=20 \times[\mathrm{NII}] \lambda 6584$

at low $[\mathrm{N}$ II $] \lambda 6584 / \mathrm{H} \beta \leq 0.1$. The determination of the $[\mathrm{O}$ II] $\lambda 3727$ flux for higher $[\mathrm{N} \mathrm{II}] \lambda 6584 / \mathrm{H} \beta$ is subject to much larger uncertainties and the use of Eq. (3) would in general result in overestimation of the [O II] $\lambda 3727$ flux and correspondingly in overestimation of the oxygen abundance. We applied this relation for selection of extremely low-metallicity candidates keeping in mind that the oxygen abundance in selected SFGs with [N II] $] 6584 / \mathrm{H} \beta=0.1-0.2$ might be overestimated. Nevertheless, additional spectroscopic observations of selected galaxies including the $[\mathrm{O}$ II $] \lambda 3727 \AA$ emission line are needed to verify and improve our indirect determination of its flux.

Three methods are used to derive the oxygen abundances. First is the direct method using prescriptions by Izotov et al. (2006a). A two-zone photoionised $\mathrm{H}$ II region model was adopted: a high-ionisation zone with temperature $T_{\mathrm{e}}(\mathrm{O}$ III), where [O III] lines originate, and a low-ionisation zone with temperature $T_{\mathrm{e}}\left(\mathrm{O}\right.$ II), where the [O II] lines originate. For $T_{\mathrm{e}}(\mathrm{O}$ II $)$, the relation between the electron temperatures $T_{\mathrm{e}}(\mathrm{O}$ III $)$ and $T_{\mathrm{e}}(\mathrm{O}$ II) from Izotov et al. (2006a) is used. Ionic and total oxygen abundances are derived using expressions for oxygen ionic abundances by Izotov et al. (2006a).

The second, semi-empirical method proposed by Izotov \& Thuan (2007) is based on the determination of the electron temperature from the strong oxygen emission lines in the galaxies with undetected [O III] $\lambda 4363 \AA$ emission line, while ionic and total oxygen abundances are derived in the way used by the direct method.

Finally, the third method is the strong-line method (Eq. (1)) developed in this paper. This simplest method is purely empirical and does not require determination of physical conditions in the $\mathrm{H}$ II region, at variance with the two other methods.

We selected 66 extremely low-metallicity SFGs in the entire SDSS spectroscopic data base for which at least one of the three values of $12+\log \mathrm{O} / \mathrm{H}$ derived by each of three methods is less than 7.4. The list of these SFGs is shown in Table A.1 and 

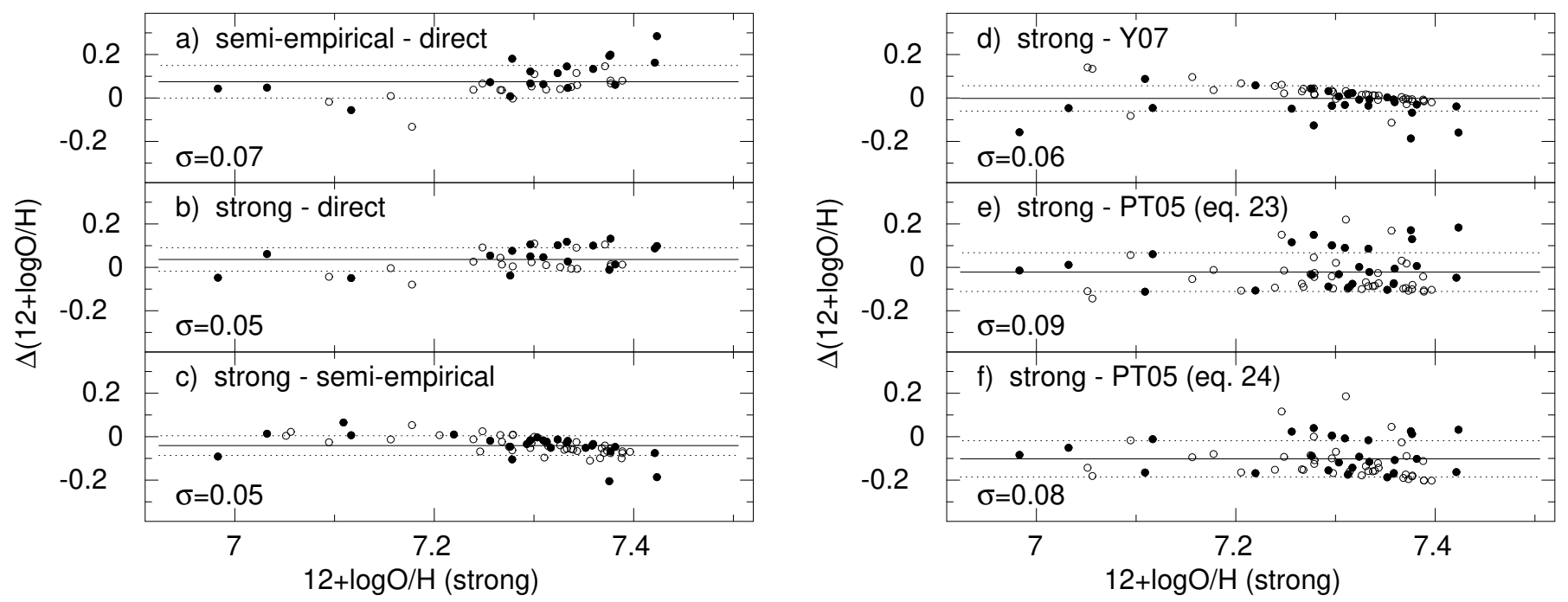

Fig. 3. Panel $a$ : distribution of differences between oxygen abundances $12+\log \mathrm{O} / \mathrm{H}$ derived by the semi-empirical and the direct methods for the sample from Table A.2 excluding galaxies with non-detected [O III] $\lambda 4363 \AA$ emission lines. Panel $b$ : as in panel a but for differences between $12+\log \mathrm{O} / \mathrm{H}$ derived by our new strong-line and the direct methods. Panel $c$ : as in panel a but for differences between $12+\log \mathrm{O} / \mathrm{H}$ derived by the strong-line and the semi-empirical methods for all SFGs from Table A.2. Panel d: as in panel c but for differences between 12+log O/H derived by the strong-line method and the strong-line method by Yin et al. (2007). Panel $e$ : as in panel $\mathrm{c}$ but for differences between $12+\log \mathrm{O} / \mathrm{H}$ derived by the strong-line method and the P-method (Eq. (23) in Pilyugin \& Thuan 2005). Panel f: as in panel c but for differences between $12+\log \mathrm{O} / \mathrm{H}$ derived by the strong-line method and the P-method (Eq. (24) in Pilyugin \& Thuan 2005). In all panels, galaxies with observed and non-observed $[\mathrm{O}$ II] $] 3727$ emission line are shown by filled and open circles, respectively. Solid horizontal lines indicate average abundance differences and dotted horizontal lines indicate $\pm 1 \sigma$ dispersions of the sample around the average values.

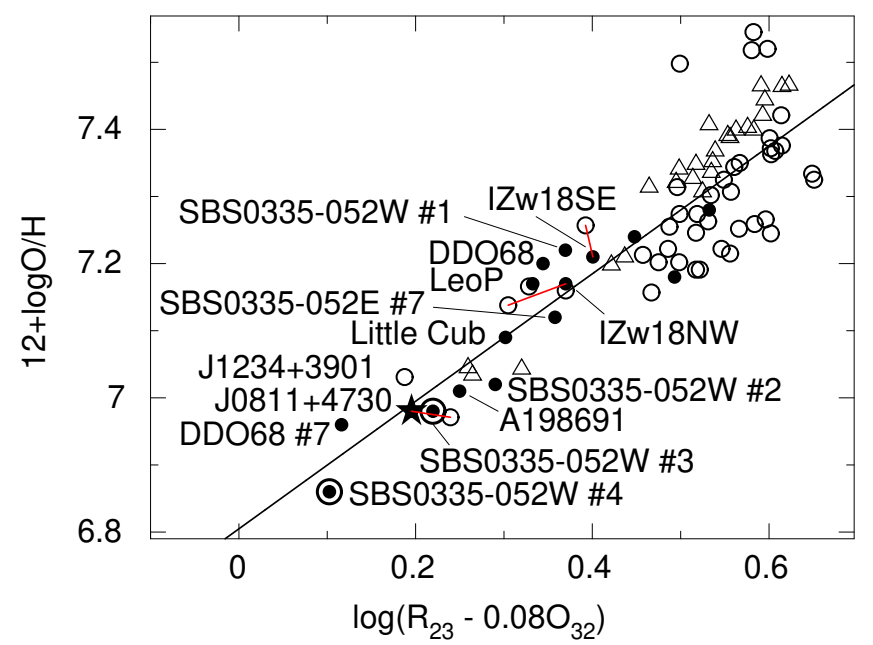

Fig. 4. $\log \left(\mathrm{R}_{23}-0.08 \mathrm{O}_{32}\right)-(12+\log \mathrm{O} / \mathrm{H})$ relation for $\mathrm{SFGs}$ with $12+\log \mathrm{O} / \mathrm{H} \leq 7.4$ from Table A.2. The SFGs from the SDSS with $12+\log \mathrm{O} / \mathrm{H}$ derived by our newly introduced direct method and by the semi-empirical method are shown by open circles and open triangles, respectively. Other symbols and the solid line are the same as in Fig. 1b. Solid lines connect different observations of the same object. Some extremely low-metallicity SFGs from the literature are labelled.

oxygen abundances derived by all three methods to check their mutual consistency are presented in Table A.2. The tables also include coordinates, redshifts, line intensities, equivalent widths of the $\mathrm{H} \beta$ emission line, and integrated characteristics, such as SDSS absolute magnitudes $M_{g}$ in the $g$-band and stellar masses $M_{\star}$ derived from the fitting of SDSS spectra. The selected galaxies are located mainly at very low redshifts, have faint absolute magnitudes and low stellar masses, characterising them as dwarf star-forming galaxies. The [O III $] \lambda 4363 \AA$ emission line

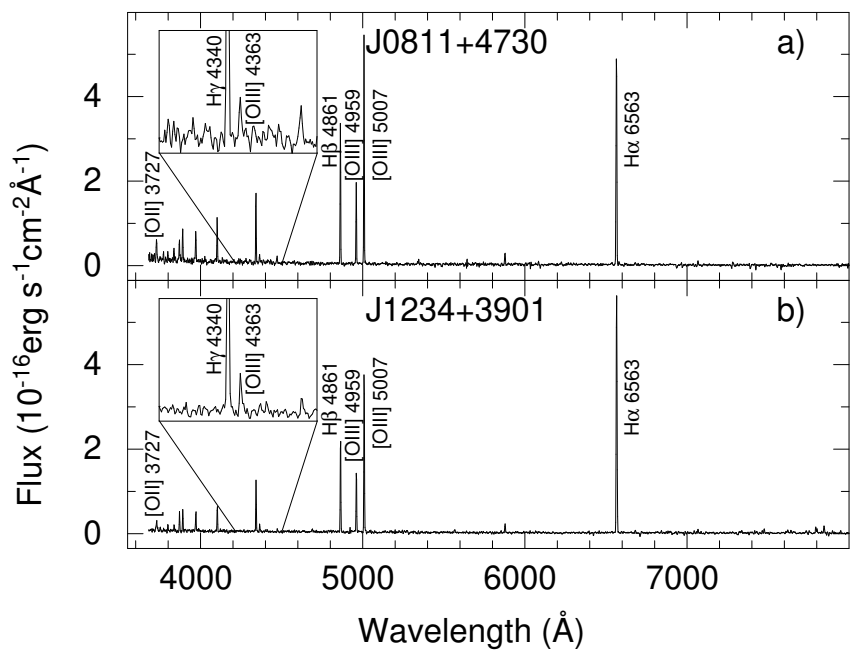

Fig. 5. SDSS spectra of the two lowest-metallicity galaxies represented in Table A.2. Insets in both panels show expanded segments of the spectra for a better view of the [O III] $\lambda 4363 \AA$ emission lines.

is detected in most selected galaxies allowing the $12+\log \mathrm{O} / \mathrm{H}$ determination by the direct method.

A notable feature is that all three methods give consistent results with a dispersion less than 0.1 dex in most cases (Table A.2). In Fig. 3 we compare oxygen abundances derived by our strong-line method with those derived by the direct, semiempirical, and some other strong-line methods in the literature. The agreement between $12+\log \mathrm{O} / \mathrm{H}$ derived with the direct method and that derived with our newly developed strong-line method is somewhat better than that derived with the semiempirical method. This is more clearly seen in Figs. 3a and b, in which we show only galaxies with detected [O III] $\lambda 4363 \AA$ emission line. On average, the oxygen abundances derived by the 
strong-line and semi-empirical methods are respectively by $\sim 0.04$ dex and $\sim 0.08$ dex higher than those derived by the direct method. In Fig. 3c we compare oxygen abundances derived by the strong-line and the semi-empirical methods which on average are consistent within $\sim 0.05$ dex. The strong-line method developed in this paper and semi-empirical method are likely more preferable compared to the direct method for galaxies with weak [O III] $] 4363 \AA$ emission, detected with poor signalto-noise ratio.

In Figs. 3d-3f we compare our strong-line method with some previously proposed strong-line methods from the literature. Yin et al. (2007) presented a simple relation between $12+\log \mathrm{O} / \mathrm{H}$ and $\mathrm{R}_{23}$ which on average gives oxygen abundances consistent with our strong-line method (Fig. 3d). However, at lowest $12+\log \mathrm{O} / \mathrm{H} \lesssim 7.1$, the relation by Yin et al. (2007) predicts oxygen abundances by as much as $\sim 0.2$ higher than those obtained with our strong-line method. Pilyugin \& Thuan (2005) develop their strong-line method by introducing the parameter $\mathrm{P}=[\mathrm{O}$ III $] \lambda 5007 /([\mathrm{O}$ II $] \lambda 3727+[\mathrm{O}$ III $] \lambda 5007)$ which takes into account the dependence on the ionization parameter. They present two relations for their method which we compare in Figs. $3 \mathrm{e}$ and $3 \mathrm{f}$ with our strong-line method. Two features of this comparison are worth to note: 1) a larger dispersion of objects compared to that in Fig. $3 \mathrm{c}$ and 2) a systematic offset of average values of $\Delta(12+\log \mathrm{O} / \mathrm{H})$ from the zero value indicating that the two modifications of the P-method give systematically higher $12+\log \mathrm{O} / \mathrm{H}$, by $\sim 0.02$ (Fig. 3e) and by $\sim 0.10$ (Fig. 3f). Finally, we note that there is no difference between the galaxies with observed and non-observed [O II] $\lambda 3727$ emission line in all panels of Fig. 3 (filled and open circles, respectively) implying reliability of calculated intensities of this line. From the above comparison we conclude that our simple strong-line method is likely the most reliable for the oxygen abundance determination of extremely low-metallicity galaxies.

The lowest-metallicity segment of the $\log \left(\mathrm{R}_{23}-0.08 \times \mathrm{O}_{32}\right)-$ $12+\log \mathrm{O} / \mathrm{H}$ relation with 66 selected extremely low-metallicity SDSS SFGs (open circles and open triangles) and some wellstudied galaxies from the literature (filled symbols) is shown in Fig. 4. Some well-known, extremely low-metallicity SFGs are labelled in the figure. The oxygen abundances for all galaxies shown in the Figure are derived by 1) the direct method if the [O III] $] \lambda 4363 \AA$ emission line is detected (open circles) or by the 2) semi-empirical method otherwise (open triangles) while the relation for the strong-line method determined by Eq. (1) is shown by the solid line. Thirty six SDSS galaxies shown in Table A. 2 were previously recovered by our team (e.g. Guseva et al. 2015, 2017; Izotov \& Thuan 2007; Izotov et al. 2012, 2018a). Three objects, I Zw 18SE, I Zw 18NW, and $\mathrm{J} 0811+4730$, are presented in both the SDSS and comparison samples and connected by solid red lines.

It is seen in Fig. 4 that selected SDSS galaxies (open circles and open triangles) are evenly distributed around the solid line implying that our newly proposed strong-line method (Eq. (1)) reasonably well reproduces galaxy metallicities. A considerable number of SFGs have $12+\log \mathrm{O} / \mathrm{H}$ lower than in the prototypical galaxy I Zw 18. Two of these galaxies, J0811+4730 (see also Sect. 3) and J1234+3901 are very close to or below the luminosity-weighted value $12+\log \mathrm{O} / \mathrm{H}=7.0$, representing the lowest-metallicity galaxies known so far. In fact, using Large Binocular Telescope observations that have high signal-tonoise ratio Izotov et al. (2018a) have confirmed that J0811+4730 has the lowest luminosity-weighted metallicity among all lowredshift SFGs with $z \lesssim 0.1$ (Izotov et al. 2018a). We note that metallicities in regions 3 and 4 of the galaxy SBS 0335-052W are even lower (Izotov et al. 2009). However, the [O III] $\lambda 4363 \AA$ emission line is not detected in spectra of these regions and their oxygen abundances are derived by the semi-empirical method. Furthermore, two other brighter regions 2 and 1 of SBS 0335-052W have higher oxygen abundances (Fig. 4) indicating an abundance gradient in this galaxy. This gradient results in a higher luminosity-weighted oxygen abundance of 7.12 averaged over the entire galaxy SBS 0335-052W. Annibali (2019) reported the very low oxygen abundance $12+\log \mathrm{O} / \mathrm{H}=6.96 \pm$ 0.09 in the $\mathrm{H}$ II region \#7 of DDO 68 derived by the direct method. However, the oxygen abundance in this region is derived with low accuracy and needs to be confirmed with higher precision. Furthermore, oxygen abundances in other $\mathrm{H}$ II regions of DDO 68 are considerably higher than in the region \#7 (Pustilnik et al. 2005; Izotov \& Thuan 2007, 2009). We note that spectra of all these regions are not present in the SDSS data base.

One of the lowest-metallicity SFGs among selected SDSS galaxies is $\mathrm{J} 1234+3901$. A single high-excitation $\mathrm{H}$ II region is observed in this galaxy with an oxygen abundance of 6.98 and 7.03 derived by the strong and direct methods, respectively. The SDSS spectra of this galaxy and another lowest-metallicity galaxy J0811+4730 from Table A.2 are shown in Fig. 5. The [O III] $\lambda 4363 \AA$ emission line is clearly detected in both spectra allowing the oxygen abundance determination by the direct method. The galaxy J1234+3901 at the redshift of 0.1330 is one of the most distant and luminous galaxies in Table A.2. Additional observations with higher signal-to-noise ratio are needed to confirm the extremely low metallicity of this galaxy.

\section{Diagnostic diagrams}

Izotov et al. (2012, 2018a) and Guseva et al. (2017) proposed to use the Baldwin, Phillips and Terlevich (BPT; Baldwin et al. 1981) diagnostic diagram [O III] $] 25007 / \mathrm{H} \beta$ vs. [N II] $] 6584 / \mathrm{H} \alpha$ for the selection of the extremely low-metallicity SFGs. They have shown that these galaxies are located in the lower-left corner of the diagram far from the main sequence of SFGs in the region defined by relations $[\mathrm{O}$ III $] \lambda 5007 / \mathrm{H} \beta<4$ and $[\mathrm{N}$ II $] \lambda 6584 / \mathrm{H} \alpha<0.03$ corresponding to $[\mathrm{N}$ II $] \lambda 6584 / \mathrm{H} \beta<0.1$. However, the use of this diagram is limited because of the weakness of the $\left[\mathrm{N}_{\mathrm{II}}\right] \lambda 6584 \AA$ emission line. In many galaxies this line is not detected. Alternatively, the diagram [O III] $] 25007 / \mathrm{H} \beta$ vs. [O II] $] 33727 / \mathrm{H} \beta$ can be used as the [O II] $] 33727 \AA$ emission line in galaxies with low metallicities is approximately 20 times brighter than the $[\mathrm{N}$ II] $\lambda 6584 \AA$ emission line.

We note that the $[\mathrm{O} \mathrm{II}] \lambda 3727 / \mathrm{H} \beta$ ratio is sensitive to dust extinction. However, in low-metallicity galaxies the internal extinction is low. It is derived from the Balmer hydrogen decrement after correction of emission line intensities for the Milky Way extinction. Both extinctions are applied to obtain extinctioncorrected line intensities in the [O III $] \lambda 5007 / \mathrm{H} \beta-\left[\mathrm{O}_{\text {II }}\right] \lambda 3727 / \mathrm{H} \beta$ diagram. We show this diagram in Fig. 6a. The shaded region indicates the location of lowest-metallicity galaxies with $12+$ $\log \mathrm{O} / \mathrm{H} \leq 7.35$. Dashed lines indicate the lines of equal $\mathrm{R}_{23}-$ $0.08 \mathrm{O}_{32}$ corresponding to equal metallicities according to Eq. (1). The SFGs selected in this paper and shown in Table A.2 are represented in the Figure by open circles and open triangles and are located in the shaded region. The range of [O II] $\lambda 3727 / \mathrm{H} \beta<2$ is somewhat larger than $\lesssim 1$ tested by galaxies with $12+\log \mathrm{O} / \mathrm{H}$ derived by the direct method (filled symbols in Fig. 6a). Therefore, metallicities in galaxies with $[\mathrm{O}$ II $] \lambda 3727 / \mathrm{H} \beta=1-2$ need to be confirmed with the direct method. 

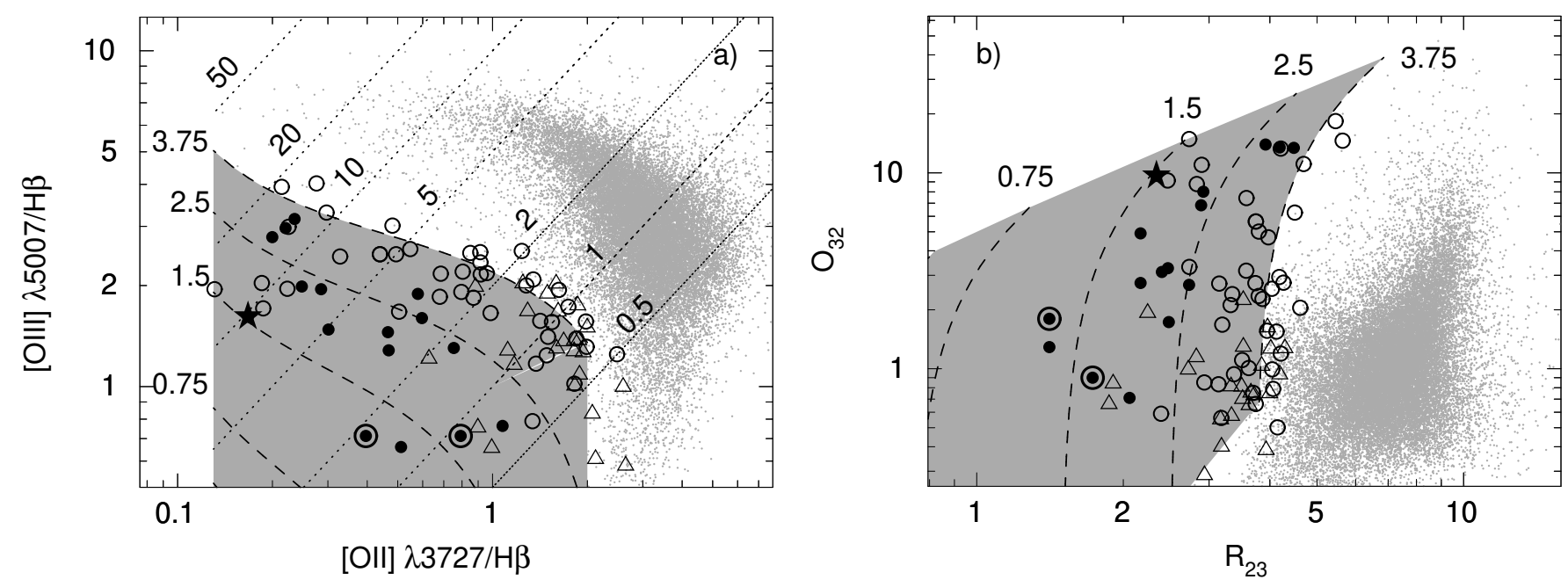

Fig. 6. Panel a: diagnostic diagram $[\mathrm{O}$ III $] \lambda 5007 / \mathrm{H} \beta-[\mathrm{O}$ II $] \lambda 3727 / \mathrm{H} \beta$. Open circles and open triangles are the extremely low-metallicity SFG candidates selected in this paper with oxygen abundances derived by the direct and semi-empirical methods, respectively. Other symbols are the same as in Fig. 1. The shaded region indicates the location of the extremely low-metallicity SFGs with $12+\log \mathrm{O} / \mathrm{H}$ in the range 6.62-7.39. Dotted and dashed lines are the lines of constant $\mathrm{O}_{32}$ and of constant $\mathrm{R}_{23}-0.08 \mathrm{O}_{32}$ (or constant $12+\log \mathrm{O} / \mathrm{H}$ ), respectively, labelled with their values. The values $\mathrm{R}_{23}-0.08 \mathrm{O}_{32}=0.75,1.5,2.5$, and 3.75 correspond to oxygen abundances $12+\log \mathrm{O} / \mathrm{H}=6.69,6.97,7.18$, and 7.35, respectively. Panel b: diagram $\mathrm{O}_{32}-\mathrm{R}_{23}$. Symbols, lines, labels and shaded region follow the definitions provided for panel a.

Additionally, for selection of extremely low-metallicity galaxies, the $\mathrm{O}_{32}-\mathrm{R}_{23}$ diagram can be used, as proposed by Izotov et al. (2018a). This diagram for selected SDSS galaxies (open circles and open triangles) and galaxies from the comparison sample (filled symbols) is shown in Fig. 6b. Similar to the diagram in Fig. 6a these galaxies are located far from the mainsequence SFGs (grey dots). We note a wide range of $\mathrm{O}_{32}$ with the highest values reaching values up to $\sim 15$. However, most of the extremely low-metallicity SFGs have $\mathrm{O}_{32}<3$. The shaded region corresponds to that in Fig. 6a. It indicates the location of extremely low-metallicity SFGs with $12+\log \mathrm{O} / \mathrm{H} \leq 7.35$.

\section{Conclusions}

We present results of a search for the extremely low-metallicity star-forming galaxies (SFGs) from the Data Release 14 of the Sloan Digital Sky Survey (SDSS DR14). Our main results are as follows.

1. A new, simple, purely empirical strong-line method based on the [O II] $\lambda 3727 \AA$ and [O III] $\lambda 4959 \AA$ A , $\lambda 5007 \AA$ emission lines is developed for the oxygen abundance determination at low metallicities and to search for such extremely lowmetallicity galaxies. This method is a modification of the well-known $\mathrm{R}_{23}$ method and takes into account the dependence of oxygen emission-line fluxes not only on the metallicity but also on the ionization parameter in the $\mathrm{H}$ II region. We adopt that the $\mathrm{O}_{32}=\left[\mathrm{O}_{\mathrm{III}}\right] \lambda 5007 /\left[\mathrm{O}_{\mathrm{II}}\right] \lambda 3727$ emissionline ratio serves as a measure of the ionization parameter. This method was calibrated using observations with high signal-to-noise ratios (S/N) of a large sample of SDSS galaxies with detected [O III] $\lambda 4363 \AA$ emission applying the direct method in combination with other samples of SFGs with reliably derived oxygen abundances via the direct method.

2. Several selection criteria were applied to build a sample of the extremely low-metallicity SFGs. First, the SDSS galaxies were preselected using a criterion $\left[\mathrm{N}_{\mathrm{II}}\right] \lambda 6584 / \mathrm{H} \beta \leq 0.2$. This allows us to exclude high-metallicity objects located on the upper branch of the $\mathrm{R}_{23}-12+\log \mathrm{O} / \mathrm{H}$ diagram. Then, all SFGs with [O III] $] 44959 / \mathrm{H} \beta \leq 0.2$ were excluded because the
[O III] $\lambda 4363 \AA$ emission line was not detected in these SFGs and thus their abundances derived by the strong method were not tested by the direct method. Finally, the remaining SFGs satisfying condition $\mathrm{R}_{23}-0.08 \mathrm{O}_{32} \lesssim 4$, corresponding to $12+\log \mathrm{O} / \mathrm{H}<7.4$, were included in the final list of candidates to the lowest-metallicity SFGs.

3. We selected 66 emission-line galaxies with $12+\log \mathrm{O} / \mathrm{H} \leq$ 7.35 in the entire SDSS DR14 using a new strong-line method. Some selected galaxies have $12+\log \mathrm{O} / \mathrm{H}$ as low as 7.0 and therefore belong to the lowest-metallicity SFGs known so far. Results of the metallicity determination by the new strong-line method are compared with those derived by a semi-empirical method (Izotov \& Thuan 2007), by a direct method, and by some strong-line methods from the literature. We find a good agreement between $12+\log \mathrm{O} / \mathrm{H}$ derived by all three first methods with deviations less than $0.1 \mathrm{dex}$, on average. The agreement with some methods selected from the literature is not as good. However, the SDSS spectra of many selected SFGs have relatively low S/N. Furthermore, in 61 percent of SFGs the [O II] $\lambda 3727 \AA$ emission line is outside the wavelength range of SDSS spectra because of their low redshifts $z \lesssim 0.02$. Therefore, its fluxes were estimated from the fluxes of weak [O II] $\lambda 7320 \AA, 7330 \AA$ or $[\mathrm{N}$ II $] \lambda 6584 \AA$ emission lines. We conclude that new observations with higher $\mathrm{S} / \mathrm{N}$ and including [O II] $\lambda 3727 \AA$ emission line are needed to confirm the low metallicity of these selected galaxies.

4. We propose the use of two diagnostic diagrams, $[\mathrm{O}$ III $] \lambda 5007 / \mathrm{H} \beta-[\mathrm{O}$ II $] \lambda 3727 / \mathrm{H} \beta$ and $\mathrm{O}_{32}-\left(\mathrm{R}_{23}-0.08 \mathrm{O}_{32}\right)$ for the selection of the extremely low-metallicity SFGs.

Acknowledgements. Y.I.I. and N.G.G. thank the hospitality of the Max-Planck Institute for Radioastronomy, Bonn, Germany. They acknowledge support from the Program of Fundamental Research of the Department of Physics and Astronomy (Project No. 0117U000240) of the National Academy of Sciences of Ukraine. Funding for the Sloan Digital Sky Survey (SDSS) has been provided by the Alfred P. Sloan Foundation, the Participating Institutions, the Nationa Aeronautics and Space Administration, the National Science Foundation, the U.S. Department of Energy, the Japanese Monbukagakusho, and the Max Planck Society. The SDSS Web site is http://www.sdss.org/. The SDSS is 
managed by the Astrophysical Research Consortium (ARC) for the Participating Institutions. The Participating Institutions are The University of Chicago, Fermilab, the Institute for Advanced Study, the Japan Participation Group, The Johns Hopkins University, Los Alamos National Laboratory, the Max-Planck-Institute for Astronomy (MPIA), the Max-Planck-Institute for Astrophysics (MPA), New Mexico State University, University of Pittsburgh, Princeton University, the United States Naval Observatory, and the University of Washington.

\section{References}

Abolfathi, B., Aguado, D. S., Aguilar, G., et al. 2018, ApJS, 235, 42 Aller, L. H. 1984, Physics of Thermal Gaseous Nebulae (Dordrecht: Reidel) Annibali, F., La Torre, V., Tosi, M., et al. 2019, MNRAS, 482, 3892 Baldwin, J. A., Phillips, M. M., \& Terlevich, R. 1981, PASP, 93, 5

Bouwens, R. J., Illingworth, G. D., Oesch, P. A., et al. 2015, ApJ, 811, 140

Cardelli, J. A., Clayton, G. C., \& Mathis, J. S. 1989, ApJ, 345, 245

Edmunds, M. G., \& Pagel, B. E. J. 1984, MNRAS, 211, 507

Ekta, B., \& Chengalur, J. N. 2010, MNRAS, 406, 1238

Ferland, G. J., Korista, K. T., Verner, D. A., et al. 1998, PASP, 110, 761

Ferland, G. J., Porter, R. L., van Hoof, P. A. M., et al. 2013, Rev. Mex. Astron. Astrofis., 49, 137

Guseva, N. G., Izotov, Y. I., Fricke, K. J., \& Henkel, C. 2015, A\&A, 579, 11

Guseva, N. G., Izotov, Y. I., Fricke, K. J., \& Henkel, C. 2017, A\&A, 599, 65

Hirschauer, A. S., Salzer, J. J., Skillman, E. D., et al. 2016, ApJ, 822, 13

Hsyu, T., Cooke, R. J., Prochaska, J. X., \& Bolte, M. 2017, ApJ, 845, L22

Izotov, Y. I., \& Thuan, T. X. 2007, ApJ, 665, 1115

Izotov, Y. I., \& Thuan, T. X. 2009, ApJ, 690, 1797

Izotov, Y. I., Thuan, T. X., \& Lipovetsky, V. A. 1994, ApJ, 435, 647

Izotov, Y. I., Stasińska, G., Meynet, G., Guseva, N. G., \& Thuan, T. X. 2006a, A\&A, 448, 955

Izotov, Y. I., Papaderos, P., Guseva, N. G., Fricke, K. J., \& Thuan, T. X. 2006b, A\&A, 454, 137
Izotov, Y. I., Guseva, N. G., Fricke, K. J., \& Papaderos, P. 2009, A\&A, 503, 61 Izotov, Y. I., Thuan, T. X., \& Guseva, N. G. 2012, A\&A, 546, 122

Izotov, Y. I., Guseva, N. G., Fricke, K. J., \& Henkel, C. 2014a, A\&A, 561, 33

Izotov, Y. I., Thuan, T. X., \& Guseva, N. G. 2014b, MNRAS, 445, 778

Izotov, Y. I., Thuan, T. X., \& Guseva, N. G. 2017, MNRAS, 471, 548

Izotov, Y. I., Thuan, T. X., Guseva, N. G., \& Liss, S. E. 2018a, MNRAS, 473 , 1956

Izotov, Y. I., Worseck, G., Schaerer, D., et al. 2018b, MNRAS, 478, 4851

Khaire, V., Srianand, R., Choudhury, T. R., \& Gaikwad, P. 2016, MNRAS, 457, 4051

Kniazev, A. Y., Egorova, E. S., \& Pustilnik, S. A. 2018, MNRAS, 479, 3842

McGaugh, S. S. 1991, ApJ, 380, 140

Nagao, T., Maiolino, R., \& Marconi, A. 2006, A\&A, 459, 85

Ouchi, M., Mobasher, B., Shimasaku, K., et al. 2009, ApJ, 706, 1136

Pagel, B. E. J., Edmunds, M. G., \& Smith, G. 1980, MNRAS, 193, 219

Papaderos, P., Guseva, N. G., Izotov, Y. I., \& Fricke, K. J. 2008, A\&A, 491, 113

Pettini, M., \& Pagel, B. E. J. 2004, MNRAS, 348, L59

Pilyugin, L. S. 2000, A\&A, 362, 325

Pilyugin, L. S., \& Grebel, E. K. 2016, MNRAS, 457, 3678

Pilyugin, L. S., \& Thuan, T. X. 2005, ApJ, 631, 231

Planck Collaboration XV. 2014, A\&A, 571, A16

Pustilnik, S. A., Kniazev, A. Y., \& Pramskij, A. G. 2005, A\&A, 443, 91

Pustilnik, S. A., Perepelitsyna, Y. A., \& Kniazev, A. Y. 2016, MNRAS, 463, 670

Robertson, B. E., Furlanetto, S. R., Schneider, E., et al. 2013, ApJ, 768, 71

Robertson, B. E., Ellis, R. S., Furlanetto, S. R., \& Dunlop, J. S. 2015, ApJ, 802, L19

Skillman, E. D. 1989, ApJ, 347, 883

Wise, J. H., \& Cen, R. 2009, ApJ, 693, 984

Wright, E. L. 2006, PASP, 118, 1711

van Zee, L., Salzer, J. J., Haynes, M. P., O’Donoghue, A. A., \& Balonek, T. J. 1998, AJ, 116, 2805

Yin, S. Y., Liang, Y. C., Hammer, F., et al. 2007, A\&A, 462, 535 
Y. I. Izotov et al.: The lowest-metallicity SDSS DR14 galaxies

\section{Appendix A: Selected extremely low-metallicity galaxies}

Table A.1. List of the extremely low-metallicity SDSS galaxies from the DR14.

\begin{tabular}{|c|c|c|c|c|c|c|c|c|c|}
\hline \multirow[t]{2}{*}{ Name } & \multicolumn{2}{|c|}{ Coordinates (J2000.0) } & \multirow[t]{2}{*}{$z^{a}$} & \multirow[t]{2}{*}{$g^{b}$} & \multirow[t]{2}{*}{ Name } & \multicolumn{2}{|c|}{ Coordinates (J2000.0) } & \multirow[t]{2}{*}{$z^{a}$} & \multirow[t]{2}{*}{$g^{b}$} \\
\hline & RA & Dec & & & & RA & Dec & & \\
\hline $\mathrm{J} 0006+2413$ & 00:06:48.96 & $+24: 13: 06.94$ & 0.03418 & 21.66 & $\mathrm{~J} 1034+1546$ & $10: 34: 05.40$ & $+15: 46: 50.14$ & 0.00410 & 17.64 \\
\hline J0015+0104 & $00: 15: 20.68$ & $+01: 04: 36.99$ & 0.00686 & 19.91 & $\mathrm{~J} 1036+2036$ & $10: 36: 39.47$ & $+20: 36: 15.80$ & 0.05487 & 21.60 \\
\hline J0029-0025 & $00: 29: 49.50$ & $-00: 25: 39.89$ & 0.01412 & 20.23 & $\mathrm{~J} 1053+4713$ & $10: 53: 21.33$ & $+47: 13: 20.85$ & 0.06796 & 21.84 \\
\hline J0042+3247 & $00: 42: 33.37$ & $+32: 47: 21.01$ & 0.14264 & 22.13 & $\mathrm{~J} 1109+2007$ & 11:09:09.53 & $+20: 07: 29.75$ & 0.00376 & 17.23 \\
\hline $\mathrm{J} 0106+2345$ & 01:06:09.17 & $+23: 45: 33.66$ & 0.05206 & 21.93 & $\mathrm{~J} 1119+0935$ & 11:19:28.09 & $+09: 35: 44.28$ & 0.00360 & 16.43 \\
\hline $\mathrm{J} 0107+0103$ & 01:07:46.56 & $+01: 03: 52.06$ & 0.00220 & 19.93 & $\mathrm{~J} 1121+3744$ & $11: 21: 46.68$ & $+37: 44: 21.18$ & 0.00644 & 17.85 \\
\hline $\mathrm{J} 0113+0052$ & $01: 13: 40.44$ & $+00: 52: 39.15$ & 0.00381 & 21.18 & $\mathrm{~J} 1139+1917$ & $11: 39: 31.78$ & $+19: 17: 24.92$ & 0.01145 & 17.69 \\
\hline $\mathrm{J} 0122+0048$ & 01:22:41.61 & $+00: 48: 42.00$ & 0.05731 & 21.62 & $\mathrm{~J} 1153+3419$ & $11: 53: 28.19$ & $+34: 19: 21.89$ & 0.00746 & 18.01 \\
\hline $\mathrm{J} 0137+2032$ & $01: 37: 40.98$ & $+20: 32: 46.50$ & 0.03480 & 22.25 & $\mathrm{~J} 1157+1713$ & $11: 57: 44.11$ & $+17: 13: 29.19$ & 0.01267 & 17.81 \\
\hline J0143+1958 & 01:43:15.15 & $+19: 58: 06.10$ & 0.00170 & 21.76 & $\mathrm{~J} 1157+5638$ & $11: 57: 54.18$ & $+56: 38: 16.71$ & 0.00150 & 16.89 \\
\hline J0153+0104 & 01:53:11.96 & $+01: 04: 40.10$ & 0.06332 & 21.52 & $\mathrm{~J} 1206+5007$ & 12:06:08.53 & $+50: 07: 21.17$ & 0.05137 & 21.76 \\
\hline J0207-0821 & 02:07:24.77 & $-08: 21: 43.60$ & 0.01267 & 20.22 & $\mathrm{~J} 1208+3727$ & 12:08:09.75 & $+37: 27: 24.65$ & 0.00356 & 17.82 \\
\hline J0222-0935 & $02: 22: 38.55$ & $-09: 35: 35.20$ & 0.11477 & 21.61 & $\mathrm{~J} 1220+4915$ & 12:20:51.61 & $+49: 15: 55.48$ & 0.01226 & 20.88 \\
\hline J0223-0918 & $02: 23: 02.68$ & $-09: 18: 22.40$ & 0.05032 & 20.99 & $\mathrm{~J} 1223+0727$ & $12: 23: 58.20$ & $+07: 27: 01.73$ & 0.00412 & 17.90 \\
\hline J0247-0404 & $02: 47: 12.80$ & $-04: 04: 31.36$ & 0.03495 & 22.07 & $\mathrm{~J} 1226+0952$ & $12: 26: 55.72$ & $+09: 52: 56.27$ & 0.00330 & 16.93 \\
\hline J0314-0108 & 03:14:26.11 & $-01: 08: 46.55$ & 0.02741 & 17.70 & $\mathrm{~J} 1228-0125$ & $12: 28: 45.54$ & $-01: 25: 26.90$ & 0.07281 & 22.09 \\
\hline J0808+3244 & 08:08:56.34 & $+32: 44: 19.20$ & 0.14640 & 20.60 & $\mathrm{~J} 1234+3901$ & $12: 34: 15.70$ & $+39: 01: 16.41$ & 0.13297 & 21.92 \\
\hline $\mathrm{J} 0811+4730$ & $08: 11: 52.12$ & $+47: 30: 26.24$ & 0.04452 & 21.32 & $\mathrm{~J} 1235+2755$ & $12: 35: 52.35$ & $+27: 55: 54.22$ & 0.00261 & 15.92 \\
\hline J0834+5905 & 08:34:37.19 & $+59: 05: 35.99$ & 0.00480 & 19.86 & $\mathrm{~J} 1244+3212$ & 12:44:11.17 & $+32: 12: 21.69$ & 0.00220 & 17.97 \\
\hline J0859+3923 & 08:59:46.93 & $+39: 23: 05.64$ & 0.00200 & 17.06 & $\mathrm{~J} 1250+1728$ & $12: 50: 31.28$ & $+17: 28: 15.95$ & 0.00290 & 16.37 \\
\hline J0906+2528 & 09:06:00.92 & $+25: 28: 11.33$ & 0.00923 & 17.41 & $\mathrm{~J} 1257+3341$ & $12: 57: 40.55$ & $+33: 41: 39.24$ & 0.00300 & 17.43 \\
\hline J0911+3135 & 09:11:59.42 & $+31: 35: 35.93$ & 0.00250 & 17.71 & $\mathrm{~J} 1258+1413$ & $12: 58: 40.20$ & $+14: 13: 00.79$ & 0.00070 & 16.20 \\
\hline J0921+4038 & $09: 21: 36.55$ & $+40: 38: 53.84$ & 0.07136 & 22.04 & $\mathrm{~J} 1308+2002$ & 13:08:28.41 & $+20: 02: 01.93$ & 0.00495 & 17.54 \\
\hline $\mathrm{J} 0934+5514 \mathrm{~A}$ & 09:34:02.03 & $+55: 14: 27.86$ & 0.00249 & 16.44 & $\mathrm{~J} 1314+2438$ & $13: 14: 59.16$ & $+24: 38: 39.15$ & 0.01265 & 18.10 \\
\hline J0934+5514B & 09:34:02.39 & $+55: 14: 23.20$ & 0.00270 & 17.58 & $\mathrm{~J} 1315+1745$ & $13: 15: 56.30$ & $+17: 45: 38.03$ & 0.00320 & 15.95 \\
\hline J0944+0936 & 09:44:44.59 & $+09: 36: 49.18$ & 0.00180 & 20.36 & $\mathrm{~J} 1414-0208$ & $14: 14: 54.13$ & $-02: 08: 22.94$ & 0.00512 & 18.00 \\
\hline J0945+3835 & $09: 45: 19.55$ & $+38: 35: 52.90$ & 0.07245 & 21.79 & $\mathrm{~J} 1424+5200$ & $14: 24: 19.49$ & $+52: 00: 38.40$ & 0.04946 & 22.64 \\
\hline J0949+3426 & 09:49:35.09 & $+34: 26: 16.36$ & 0.00500 & 22.24 & $\mathrm{~J} 1433+1544$ & $14: 33: 21.29$ & $+15: 44: 21.60$ & 0.02027 & 21.48 \\
\hline J0950+3127 & 09:50:19.49 & $+31: 27: 22.24$ & 0.00180 & 17.77 & $\mathrm{~J} 1444+4237$ & $14: 44: 12.80$ & $+42: 37: 44.01$ & 0.00211 & 21.39 \\
\hline J0955+6442 & 09:55:31.45 & $+64: 42: 50.06$ & 0.00320 & 17.92 & $\mathrm{~J} 1522+4201$ & $15: 22: 55.55$ & $+42: 01: 58.30$ & 0.00194 & 17.48 \\
\hline J0956+2849 & 09:56:46.05 & $+28: 49: 43.78$ & 0.00160 & 14.64 & $\mathrm{~J} 1640+2845$ & $16: 40: 21.43$ & $+28: 45: 55.92$ & 0.00329 & 17.00 \\
\hline J0959+4626 & 09:59:05.76 & $+46: 26: 50.49$ & 0.00200 & 17.79 & $\mathrm{~J} 1703+2126$ & 17:03:11.59 & $+21: 26: 21.20$ & 0.10171 & 20.21 \\
\hline $\mathrm{J} 1000+3032$ & 10:00:36.54 & $+30: 32: 09.78$ & 0.00170 & 17.77 & J2104-0035 & 21:04:55.31 & $-00: 35: 22.24$ & 0.00465 & 18.13 \\
\hline
\end{tabular}

Notes. ${ }^{(a)}$ Redshift. ${ }^{(b)}$ SDSS $g$-band magnitude. 
Table A.2. Emission-line intensities and oxygen abundances of the extremely low-metallicity SDSS galaxies from the DR14.

\begin{tabular}{|c|c|c|c|c|c|c|c|c|c|c|c|c|}
\hline \multirow[b]{2}{*}{ Name } & \multicolumn{5}{|c|}{ Line intensities $^{a}$} & \multirow[b]{2}{*}{$F(\mathrm{H} \beta)^{d}$} & \multirow[b]{2}{*}{$\mathrm{EW}(\mathrm{H} \beta)^{e}$} & \multicolumn{3}{|c|}{$12+\log \mathrm{O} / \mathrm{H}^{b}$} & \multirow[b]{2}{*}{$M_{g}^{f}$} & \multirow[b]{2}{*}{$\log M_{\star}^{g}$} \\
\hline & 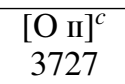 & $\begin{array}{c}{[\mathrm{O} \text { III] }} \\
4959\end{array}$ & $\begin{array}{c}{[\mathrm{O} \text { III] }} \\
4363\end{array}$ & $\begin{array}{l}{[\mathrm{O} \text { II] }} \\
7325\end{array}$ & $\begin{array}{l}{\left[\mathrm{N}_{\mathrm{II}}\right]} \\
6584\end{array}$ & & & $d$ & se & $s$ & & \\
\hline $\mathrm{J} 0006+2413$ & 0.439 & 0.826 & 0.121 & $\ldots$ & & 3.9 & 39.5 & 7.25 & 7.31 & 7.30 & -15.0 & 7.6 \\
\hline $\mathrm{J} 0015+0104^{h}$ & $(0.898)$ & 0.252 & $\ldots$ & $\ldots$ & 0.045 & 5.0 & 177.1 & & 7.03 & 7.06 & -15.4 & 4.2 \\
\hline J0029-0025 & $(0.959)$ & 0.726 & 0.146 & $\ldots$ & 0.048 & 1.8 & 35.1 & 7.25 & 7.37 & 7.34 & -14.4 & 6.3 \\
\hline $\mathrm{J} 0042+3247^{h}$ & 0.804 & 0.733 & 0.100 & & $\ldots$ & 2.4 & 68.0 & 7.22 & 7.34 & 7.32 & -17.2 & 8.0 \\
\hline $\mathrm{J} 0106+2345$ & 0.223 & 0.652 & 0.063 & . & . & 0.4 & 277.0 & 7.17 & 7.11 & 7.12 & -15.2 & 6.4 \\
\hline J0107+0103 & $(1.682)$ & 0.455 & $\ldots$ & 0.056 & 0.144 & 18.5 & 20.6 & $\ldots$ & 7.35 & 7.31 & -10.3 & 5.8 \\
\hline $\mathrm{J} 0113+0052^{h}$ & $(0.680)$ & 0.617 & 0.091 & $\ldots$ & 0.034 & 6.6 & 46.3 & 7.16 & 7.22 & 7.25 & -12.3 & 5.9 \\
\hline $\mathrm{J} 0122+0048^{h}$ & 0.548 & 0.856 & 0.100 & $\ldots$ & $\ldots$ & 7.2 & 156.0 & 7.22 & 7.36 & 7.33 & -16.1 & 6.8 \\
\hline J0137+2032 & 1.293 & 0.559 & $\ldots$ & $\ldots$ & $\ldots$ & 0.4 & 26.7 & $\ldots$ & 7.34 & 7.31 & -15.2 & 6.7 \\
\hline $\mathrm{J} 0143+1958^{h}$ & 1.597 & 0.433 & $\ldots$ & $\ldots$ & $\ldots$ & 1.4 & 20.8 & $\ldots$ & 7.33 & 7.29 & -7.8 & 4.4 \\
\hline $\mathrm{J} 0153+0104^{h}$ & 0.214 & 1.311 & 0.134 & $\ldots$ & $\ldots$ & 8.3 & 229.1 & 7.39 & 7.58 & 7.38 & -16.2 & 6.2 \\
\hline $\mathrm{J} 0207-0821^{h}$ & 0.883 & 0.661 & $\ldots$ & $\ldots$ & 0.105 & 3.7 & 29.3 & $\ldots$ & 7.31 & 7.30 & -14.2 & 6.9 \\
\hline $\mathrm{J} 0222-0935^{h}$ & 0.915 & 0.781 & 0.103 & & 0.099 & 1.9 & 59.5 & 7.26 & 7.39 & 7.36 & -17.2 & 7.9 \\
\hline $\mathrm{J} 0223-0918^{h}$ & 1.248 & 0.677 & $\ldots$ & $\ldots$ & $\ldots$ & 0.6 & 23.1 & $\ldots$ & 7.40 & 7.36 & -16.0 & 7.5 \\
\hline J0247-0404 & 0.628 & 0.404 & $\ldots$ & $\ldots$ & $\ldots$ & 3.7 & 85.0 & $\ldots$ & 7.04 & 7.11 & -14.1 & 7.0 \\
\hline J0314-0108 & 0.494 & 0.826 & 0.084 & 0.021 & 0.113 & 26.2 & 31.3 & 7.26 & 7.33 & 7.31 & -18.9 & 7.3 \\
\hline J0808+3244 & 1.819 & 0.340 & 0.036 & $\ldots$ & 0.102 & 8.6 & 50.4 & 7.31 & 7.32 & 7.28 & -18.9 & 8.9 \\
\hline $\mathrm{J} 0811+4730^{h}$ & 0.187 & 0.570 & 0.088 & $\ldots$ & & 15.5 & 282.0 & 6.97 & 7.02 & 7.03 & -17.3 & 5.8 \\
\hline $\mathrm{J} 0834+5905^{h}$ & $(0.986)$ & 0.552 & 0.059 & $\ldots$ & 0.049 & 8.6 & 22.3 & 7.22 & 7.26 & 7.27 & -15.6 & 6.7 \\
\hline $\mathrm{J} 0859+3923^{h}$ & (1.989) & 0.438 & 0.086 & 0.065 & 0.091 & 5.9 & 28.3 & 7.35 & 7.41 & 7.34 & -12.8 & 5.4 \\
\hline $\mathrm{J} 0906+2528^{h}$ & (1.864) & 0.582 & $\ldots$ & $\ldots$ & 0.093 & 7.0 & 14.4 & $\ldots$ & 7.46 & 7.39 & -16.2 & 6.7 \\
\hline $\mathrm{J} 0911+3135^{h}$ & $(1.544)$ & 0.519 & 0.058 & $\ldots$ & 0.077 & 8.4 & 16.2 & 7.32 & 7.37 & 7.33 & -12.9 & 5.6 \\
\hline J0921+4038 & 0.276 & 1.342 & 0.181 & $\ldots$ & $\ldots$ & 7.4 & 300.5 & 7.32 & 7.61 & 7.42 & -15.9 & 6.6 \\
\hline $\mathrm{J} 0934+5514 \mathrm{~A}^{h}$ & $(0.185)$ & 0.678 & 0.072 & 0.016 & 0.006 & 194.9 & 57.6 & 7.14 & 7.12 & 7.09 & -14.1 & 6.2 \\
\hline $\mathrm{J} 0934+5514 \mathrm{~B}^{h}$ & $(0.505)$ & 0.557 & 0.045 & 0.014 & 0.014 & 374.6 & 147.7 & 7.26 & 7.12 & 7.18 & -13.1 & 5.1 \\
\hline J0944+0936 & (1.844) & 0.463 & 0.050 & 0.060 & 0.160 & 7.6 & 18.7 & 7.34 & 7.39 & 7.34 & -10.5 & 5.3 \\
\hline $\mathrm{J} 0945+3835^{h}$ & 1.238 & 0.845 & 0.124 & $\ldots$ & 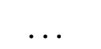 & 3.5 & 93.9 & 7.33 & 7.50 & 7.42 & -16.8 & 6.9 \\
\hline J0949+3426 & $(1.280)$ & 0.667 & 0.039 & 0.042 & 0.118 & 12.8 & 33.4 & 7.55 & 7.40 & 7.36 & -16.2 & 6.0 \\
\hline $\mathrm{J} 0950+3127^{h}$ & $(2.485)$ & 0.416 & 0.071 & $\ldots$ & 0.124 & 2.8 & 12.4 & 7.42 & 7.49 & 7.39 & -12.7 & 5.3 \\
\hline $\mathrm{J} 0955+6442^{h}$ & (1.339) & 0.263 & 0.047 & $\ldots$ & 0.067 & 6.5 & 62.9 & 7.16 & 7.17 & 7.16 & -12.9 & 5.1 \\
\hline $\mathrm{J} 0956+2849^{h}$ & (2.124) & 0.203 & $\ldots$ & 0.070 & 0.071 & 7.6 & 17.8 & $\ldots$ & 7.31 & 7.25 & -14.6 & 4.9 \\
\hline $\mathrm{J} 0959+4626^{h}$ & (1.486) & 0.413 & 0.050 & $\ldots$ & 0.074 & 9.5 & 54.1 & 7.26 & 7.29 & 7.27 & -12.2 & 5.2 \\
\hline $\mathrm{J} 1000+3032^{h}$ & (1.939) & 0.422 & $\ldots$ & $\ldots$ & 0.097 & 3.3 & 16.4 & $\ldots$ & 7.39 & 7.33 & -12.5 & 5.5 \\
\hline $\mathrm{J} 1034+1546^{h}$ & (1.979) & 0.521 & 0.057 & - & 0.099 & 9.6 & 19.8 & 7.37 & 7.45 & 7.38 & -13.9 & 6.0 \\
\hline $\mathrm{J} 1036+2036^{h}$ & 0.917 & 0.719 & 0.076 & $\ldots$ & $\ldots$ & 2.8 & 83.3 & 7.31 & 7.35 & 7.33 & -15.6 & 7.0 \\
\hline $\mathrm{J} 1053+4713$ & 0.481 & 1.005 & 0.130 & $\ldots$ & $\ldots$ & 5.2 & 92.2 & 7.24 & 7.44 & 7.38 & -16.9 & 7.3 \\
\hline $\mathrm{J} 1109+2007^{h}$ & $(0.685)$ & 0.723 & 0.093 & 0.023 & 0.056 & 15.8 & 61.5 & 7.19 & 7.30 & 7.30 & -14.1 & 6.1 \\
\hline $\mathrm{J} 1119+0935^{h}$ & $(0.870)$ & 0.613 & 0.058 & 0.029 & 0.069 & 23.2 & 29.8 & 7.27 & 7.27 & 7.28 & -14.0 & 5.9 \\
\hline $\mathrm{J} 1121+3744^{h}$ & $(1.825)$ & 0.336 & $\ldots$ & $\ldots$ & 0.091 & 2.0 & 12.9 & $\ldots$ & 7.32 & 7.28 & -15.2 & 6.4 \\
\hline J1139+1917 & $(1.821)$ & 0.459 & $\cdots$ & $\ldots$ & 0.091 & 7.1 & 39.3 & $\cdots$ & 7.39 & 7.33 & -17.1 & 7.3 \\
\hline $\mathrm{J} 1153+3419$ & $(1.876)$ & 0.459 & $\ldots$ & 0.062 & 0.183 & 4.4 & 9.0 & $\ldots$ & 7.40 & 7.34 & -15.4 & 6.9 \\
\hline $\mathrm{J} 1157+1713$ & $(0.994)$ & 0.219 & $\ldots$ & $\ldots$ & 0.050 & 2.1 & 9.9 & $\ldots$ & 7.05 & 7.05 & -17.1 & 7.2 \\
\hline $\mathrm{J} 1157+5638$ & $(0.297)$ & 1.099 & 0.076 & 0.010 & 0.030 & 52.2 & 85.1 & 7.52 & 7.47 & 7.36 & -13.3 & 5.2 \\
\hline $\mathrm{J} 1206+5007^{i}$ & 0.439 & 0.826 & 0.121 & $\ldots$ & $\ldots$ & 7.4 & 218.5 & 7.19 & 7.31 & 7.30 & -15.5 & 6.0 \\
\hline $\mathrm{J} 1206+5007^{i}$ & 0.328 & 0.813 & 0.085 & $\ldots$ & $\ldots$ & 7.5 & 195.4 & 7.20 & 7.28 & 7.26 & -15.3 & 6.7 \\
\hline $\mathrm{J} 1208+3727^{h}$ & (1.889) & 0.362 & $\ldots$ & 0.062 & 0.083 & 5.3 & 18.7 & $\ldots$ & 7.35 & 7.30 & -14.6 & 6.2 \\
\hline $\mathrm{J} 1220+4915^{h}$ & 0.225 & 0.997 & 0.128 & $\ldots$ & - & 13.9 & 169.2 & 7.20 & 7.38 & 7.28 & -13.0 & 5.6 \\
\hline $\mathrm{J} 1223+0727$ & $(1.347)$ & 0.695 & 0.065 & $\ldots$ & 0.067 & 8.7 & 26.3 & 7.36 & 7.43 & 7.38 & -13.9 & 5.8 \\
\hline
\end{tabular}

Notes. ${ }^{(a)}$ Extinction-corrected line intensity relative to the $\mathrm{H} \beta$ emission line intensity. Values in parentheses are calculated [O II] $\lambda 3727$ line intensities in galaxies where this line is outside the wavelength range of the SDSS spectrum. ${ }^{(b)} \mathrm{s}$ - strong line method, se - semi-empirical method, $\mathrm{d}$ - direct method. ${ }^{\left({ }^{c}\right)}$ The value in parentheses is derived from the [O II]7325 flux if available or from the [N $\left.\mathrm{II}\right] 6584$ flux otherwise. ${ }^{(d)} \mathrm{Observed}$ flux of the $\mathrm{H} \beta$ emission line in $10^{-16} \mathrm{erg} \mathrm{s}^{-1} \mathrm{~cm}^{-2} .{ }^{(e)}$ Equivalent width of the $\mathrm{H} \beta$ emission line in $\AA .{ }^{(f)}$ Absolute SDSS $g$ magnitude. ${ }^{(g)} \log$ of stellar mass in $\log M_{\odot} .{ }^{(h)}$ Discussed in Izotov \& Thuan (2007), Papaderos et al. (2008), Izotov et al. (2006b, 2012, 2018a), Guseva et al. (2015, 2017). ${ }^{(i)}$ Different observations of the same galaxy. 
Table A.2. continued.

\begin{tabular}{|c|c|c|c|c|c|c|c|c|c|c|c|c|}
\hline \multirow[b]{2}{*}{ Name } & \multicolumn{5}{|c|}{ Line intensities $^{a}$} & \multirow[b]{2}{*}{$F(\mathrm{H} \beta)^{d}$} & \multirow[b]{2}{*}{$\mathrm{EW}(\mathrm{H} \beta)^{e}$} & \multicolumn{3}{|c|}{$12+\log \mathrm{O} / \mathrm{H}^{b}$} & \multirow[b]{2}{*}{$M_{g}^{f}$} & \multirow[b]{2}{*}{$\log M_{\star}^{g}$} \\
\hline & $\begin{array}{c}\left.{ }^{[\mathrm{O}} \text { II }\right]^{c} \\
3727 \\
\end{array}$ & $\begin{array}{c}{\left[\mathrm{O}_{\mathrm{III}}\right]} \\
4959\end{array}$ & $\begin{array}{c}{[\mathrm{O} \text { III }]} \\
4363\end{array}$ & $\begin{array}{l}\mathrm{O} \text { II }] \\
7325\end{array}$ & $\begin{array}{l}{[\mathrm{N} \text { II }]} \\
6584\end{array}$ & & & $d$ & se & $s$ & & \\
\hline $\mathrm{J} 1226+0952$ & $(1.374)$ & 0.390 & 0.062 & 0.045 & 0.102 & 6.5 & 35.3 & 7.21 & 7.25 & 7.24 & -14.2 & 5.4 \\
\hline $\mathrm{J} 1228-0125^{h}$ & 1.616 & 0.558 & 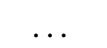 & $\ldots$ & 0.043 & 1.8 & 107.0 & & 7.40 & 7.35 & -16.4 & 6.9 \\
\hline $\mathrm{J} 1234+3901$ & 0.131 & 0.650 & 0.069 & & & 12.4 & 242.0 & 7.03 & 7.07 & 6.98 & -17.6 & 7.4 \\
\hline $\mathrm{J} 1235+2755^{h}$ & $(1.493)$ & 0.631 & $\ldots$ & 0.049 & 0.118 & 12.2 & 31.3 & $\ldots$ & 7.42 & 7.37 & -7.9 & 5.6 \\
\hline $\mathrm{J} 1244+3212^{h}$ & $(0.796)$ & 0.638 & 0.038 & 0.026 & 0.086 & 23.9 & 7.2 & 7.50 & 7.27 & 7.28 & -15.7 & 6.3 \\
\hline $\mathrm{J} 1250+1728$ & $(2.647)$ & 0.194 & $\ldots$ & 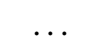 & 0.132 & 3.7 & 15.2 & 等 & 7.41 & 7.31 & -14.6 & 5.8 \\
\hline $\mathrm{J} 1257+3341^{h}$ & $(1.741)$ & 0.576 & 0.039 & 0.057 & 0.040 & 29.9 & 108.8 & 7.52 & 7.44 & 7.37 & -13.8 & 5.4 \\
\hline $\mathrm{J} 1258+1413^{h}$ & $(1.500)$ & 0.469 & 0.068 & 0.049 & 0.073 & 19.0 & 16.3 & 7.27 & 7.33 & 7.30 & -12.5 & 5.8 \\
\hline $\mathrm{J} 1308+2002^{h}$ & $(1.592)$ & 0.676 & $\ldots$ & $\ldots$ & 0.080 & 3.6 & 7.3 & & 7.47 & 7.40 & -15.5 & 6.3 \\
\hline $\mathrm{J} 1314+2438$ & $(2.074)$ & 0.277 & $\ldots$ & . & 0.104 & 1.9 & 6.2 & & 7.34 & 7.28 & -16.6 & 6.9 \\
\hline $\mathrm{J} 1315+1745$ & (1.624) & 0.647 & 0.070 & 0.053 & 0.145 & 23.3 & 14.8 & 7.38 & 7.46 & 7.39 & -15.6 & 6.4 \\
\hline $\mathrm{J} 1414-0208^{h}$ & $(1.417)$ & 0.523 & 0.051 & 0.047 & 0.025 & 11.4 & 54.9 & 7.30 & 7.34 & 7.31 & -14.2 & 6.1 \\
\hline $\mathrm{J} 1424+5200$ & 1.117 & 0.427 & $\ldots$ & $\ldots$ & 0.065 & 0.2 & 12.5 & $\ldots$ & 7.21 & 7.22 & -15.9 & 7.1 \\
\hline J1433+1544 & 0.914 & 0.838 & 0.078 & 0.040 & 0.066 & 2.0 & 34.4 & 7.37 & 7.45 & 7.39 & -13.5 & 7.1 \\
\hline $\mathrm{J} 1444+4237^{h}$ & $(0.850)$ & 0.833 & 0.100 & 0.028 & 0.049 & 17.9 & 30.2 & 7.27 & 7.41 & 7.37 & -14.4 & 5.4 \\
\hline $\mathrm{J} 1522+4201$ & $(2.598)$ & 0.333 & $\ldots$ & $\ldots$ & 0.130 & 4.0 & 18.5 & $\ldots$ & 7.47 & 7.37 & -12.3 & 5.5 \\
\hline $\mathrm{J} 1640+2845$ & (1.998) & 0.500 & $\ldots$ & $\ldots$ & 0.100 & 3.2 & 32.3 & $\ldots$ & 7.44 & 7.37 & -14.7 & 5.8 \\
\hline $\mathrm{J} 1703+2126$ & 1.816 & 0.425 & $\ldots$ & $\cdots$ & 0.077 & 0.7 & 8.9 & $\cdots$ & 7.37 & 7.32 & -19.0 & 8.6 \\
\hline $\mathrm{J} 2104-0035^{h}$ & (1.171) & 0.387 & $\ldots$ & $\ldots$ & 0.042 & 9.6 & 20.7 & $\ldots$ & 7.20 & 7.20 & -14.9 & 6.2 \\
\hline
\end{tabular}

